\title{
A comparison of combination dopamine and epinephrine treatment with high-dose dopamine alone in asphyxiated newborn piglets after resuscitation
}

\author{
Namdar Manouchehri', David L. Bigam', Thomas Churchill', David Rayner², Chloe Joynt ${ }^{3}$ and Po-Yin Cheung ${ }^{1,3}$
}

BACKGROUND: When asphyxiated neonates require additional cardiovascular support to moderate doses of dopamine infusion, controversy exists on the differential hemodynamic effects of two approaches (adding a second inotrope vs. increasing dopamine dosage). We hypothesized that high-dose dopamine (HD) would be detrimental to systemic and regional perfusion as compared with dopamine and epinephrine $(D+E)$ combination therapy using a swine model of neonatal hypoxiareoxygenation $(H-R)$.

METHODS: Twenty-seven piglets (1-4 d, 1.5-2.5 kg) were used for continuous monitoring of systemic arterial pressure (mean arterial pressure, MAP) and pulmonary arterial pressure (PAP), cardiac output (cardiac index, Cl), and carotid (carotid artery flow index, CAFI), superior mesenteric (superior mesenteric artery flow index), and renal arterial flows. $H$-R piglets underwent $2 \mathrm{~h}$ of hypoxia followed by $2 \mathrm{~h}$ of reoxygenation before drug infusion (2 h).

RESULTS: The hemodynamics of $\mathrm{H}-\mathrm{R}$ piglets deteriorated gradually after reoxygenation. HD and D + E infusions improved $\mathrm{Cl}$ similarly (both groups vs. control; $P<0.05$ ). Both regimens increased MAP $(P<0.05)$ but not PAP, with decreased PAP/MAP ratio in $D+E$ piglets. Both regimens improved CAFI and superior mesenteric artery flow index, with decreased mesenteric vascular resistance in HD-treated piglets. No significant effect on renal perfusion was observed.

CONCLUSION: In H-R newborn piglets treated with a moderate dose of dopamine, adding epinephrine or further increasing dopamine improved systemic hemodynamics similarly; these treatments have differential effects on the pulmonary and mesenteric circulations.

$T$ he World Health Organization attributes $23 \%$ of the 4 million neonatal deaths each year to asphyxia (1). Neonatal survival is challenged by asphyxia, with variable effects on different organ systems. When 72 asphyxiated neonates were followed prospectively, evidence of multiorgan dysfunction was observed, with abnormalities of the central nervous, cardiovascular, gastrointestinal, and renal systems (2).

Following asphyxia, neonates develop shock with reduced cardiac output, systemic hypotension, and pulmonary hypertension (3-5). Subsequent regional hypoperfusion compromises cerebral, gastrointestinal, and renal function. Vasoactive medications-inotropes and vasopressors-have long been used to treat shock in neonates despite a paucity of evidence on their effects, particularly with respect to organ blood flow and tissue perfusion $(6,7)$.

Dopamine, the most frequently used vasoactive agent in neonates, exerts dose-dependent $\alpha$ - and $\beta$-adrenergic and dopaminergic effects (8). Other than dopamine, epinephrine is commonly used in neonatal intensive care units. A significant improvement of cardiac function has been observed after administration of epinephrine in various asphyxiated newborn porcine models $(9,10)$. Clinically, epinephrine has been shown to be as effective as dopamine for the treatment of hypotension in low-birth-weight infants (11). Because it may exert significant adrenergic effect at low doses in the developing immature cardiovascular system, epinephrine has often been considered as an additional agent in treating neonatal hypotension refractory to initial dopamine infusion in lieu of increasing dopamine doses (12). Indeed, we previously showed that in asphyxiated newborn piglets, low-dose epinephrine $(0.2 \mathrm{mcg} /$ $\mathrm{kg} / \mathrm{min})$ added to dopamine $(10 \mathrm{mcg} / \mathrm{kg} / \mathrm{min})$ enhanced cardiac output and blood pressure to a similar extent as high-dose epinephrine ( $1 \mathrm{mcg} / \mathrm{kg} / \mathrm{min})$ alone (13). Furthermore, in the hypotensive low-birth-weight preterm population, Seri and Evans demonstrated normalized blood pressure and improved urine output with the addition of epinephrine to dopamine infusion (I. Seri and N. Evans, personal communication).

Treatment with high doses of dopamine $(\geq 20 \mathrm{mcg} / \mathrm{kg} / \mathrm{min})$ is often avoided given potentially excessive $\alpha$-adrenergic stimulation with vasoconstriction and increased afterload $(7,14)$. However, we previously demonstrated an improved mesenteric perfusion at high-dose dopamine (HD) infusion in asphyxiated newborn piglets (9). Therefore, monotherapy with HD may be an alternative approach to combination therapy of adding a second vasoactive/inotropic agent at low dose to dopamine infusion for neonates with severe cardiovascular dysfunction $(13,15,16)$. Little information is available regarding direct comparison of these two treatment strategies on systemic and regional hemodynamics. 
Table 1. Arterial blood gas and acid-base status

\begin{tabular}{lcccc}
\hline & Baseline & End-hypoxia & Predrug & End drug \\
\hline Time & $0 \mathrm{~min}$ & $120 \mathrm{~min}$ & $240 \mathrm{~min}$ & $360 \mathrm{~min}$ \\
\hline $\mathrm{pH}$ & & & & \\
Sham & $7.47 \pm 0.04$ & $7.44 \pm 0.02$ & $7.46 \pm 0.01$ & $7.45 \pm 0.02$ \\
Control & $7.45 \pm 0.02$ & $7.02 \pm 0.02^{*}$ & $7.38 \pm 0.02$ & $7.41 \pm 0.02$ \\
$\mathrm{D}+\mathrm{E}$ & $7.44 \pm 0.02$ & $7.04 \pm 0.03^{*}$ & $7.40 \pm 0.01$ & $7.36 \pm 0.02^{*}$ \\
$\mathrm{HD}$ & $7.47 \pm 0.04$ & $7.06 \pm 0.03^{*}$ & $7.39 \pm 0.04$ & $7.37 \pm 0.03^{*}$ \\
$\mathrm{HCO}(\mathrm{mmol} / \mathrm{l})$ & & & & \\
Sham & $27.1 \pm 1.9$ & $27.2 \pm 1.5$ & $28.3 \pm 1.0$ & $27.4 \pm 1.2$ \\
$\mathrm{Control}$ & $26.7 \pm 1.1$ & $9.2 \pm 0.4^{*}$ & $22.5 \pm 0.9^{*}$ & $25.0 \pm 1.4$ \\
$\mathrm{D}+\mathrm{E}$ & $26.4 \pm 4.4$ & $9.7 \pm 0.7^{*}$ & $21.5 \pm 1.2^{*}$ & $22.1 \pm 1.0^{*}$ \\
$\mathrm{HD}$ & $26.7 \pm 1.9$ & $10.5 \pm 0.9^{*}$ & $22.0 \pm 1.5^{*}$ & $22.6 \pm 1.3^{*}$ \\
PaO ${ }_{2}(\mathrm{~mm} \mathrm{Hg})$ & & & & \\
Sham & $87.1 \pm 5.8$ & $71.5 \pm 3.0$ & $70.5 \pm 2.9$ & $67.1 \pm 1.8$ \\
Control & $83.6 \pm 3.3$ & $38.3 \pm 2.2^{*}$ & $65.2 \pm 1.1$ & $70.1 \pm 2.2$ \\
$\mathrm{D}+\mathrm{E}$ & $79.6 \pm 7.7$ & $42.0 \pm 4.6^{*}$ & $68.3 \pm 6.4$ & $78.7 \pm 5.7$ \\
$\mathrm{HD}$ & $89.1 \pm 6.9$ & $39.9 \pm 4.4^{*}$ & $70.5 \pm 4.1$ & $70.1 \pm 1.3$ \\
Lactate $(\mathrm{mmol} / \mathrm{l})$ & & & \\
Sham & $3.7 \pm 0.4$ & $2.6 \pm 0.3$ & $1.8 \pm 0.3$ & $1.7 \pm 0.2$ \\
Control & $2.8 \pm 0.2$ & $13.6 \pm 1.1^{*}$ & $4.1 \pm 0.4^{*}$ & $2.0 \pm 0.1$ \\
$\mathrm{D}+\mathrm{E}$ & $3.2 \pm 0.4$ & $14.0 \pm 1.0^{*}$ & $4.7 \pm 0.8^{*}$ & $3.2 \pm 0.6$ \\
$\mathrm{HD}$ & $3.0 \pm 0.2$ & $13.0 \pm 0.8^{*}$ & $3.4 \pm 0.3^{*}$ & $1.8 \pm 0.3$ \\
\hline
\end{tabular}

Hypoxic-reoxygenated piglets treated with combination $\mathrm{D}+\mathrm{E}(n=7) ; \mathrm{HD}(n=7)$; or saline placebo (control, $n=7$ ). Sham-operated piglets had no hypoxia-reoxygenation $(n=6)$.

$\mathrm{D}+\mathrm{E}$, dopamine and epinephrine; $\mathrm{HD}$, high-dose dopamine.

${ }^{*} P<0.05$ vs. sham.

Using an established swine model of neonatal hypoxiareoxygenation (H-R), we primarily compared the efficacy of dopamine and low-dose epinephrine $(\mathrm{D}+\mathrm{E})$ combination treatment with $\mathrm{HD}$ alone on cardiac recovery after asphyxia. Other than the cardiac output, we also examined their effects on (i) carotid, mesenteric, and renal perfusion; (ii) oxygen transport; and (iii) degree of histologic injury after H-R. We hypothesized that monotherapy with HD would have similar effects on cardiac output but differential effects on regional circulation as compared with $\mathrm{D}+\mathrm{E}$ combination therapy in neonatal H-R.

\section{RESULTS}

Of the 29 piglets instrumented, two were excluded for complications related to surgery (1) and hypoxia (1), leaving 27 piglets for analysis. Animals were aged $2.2 \pm 0.2 \mathrm{~d}$ and weighed $1.9 \pm 0.1 \mathrm{~kg}$. No significant difference in gender, hemodynamic, and physiologic parameters was recorded after stabilization among experimental groups.

\section{Hypoxia}

Following $2 \mathrm{~h}$ of hypoxia $\left(\mathrm{PaO}_{2} 40 \pm 1 \mathrm{~mm} \mathrm{Hg}\right)$ (Table 1), all $\mathrm{H}-\mathrm{R}$ piglets were in cardiogenic shock, exhibiting significant hypotension (mean arterial pressure, MAP: $28 \pm 1 \mathrm{~mm} \mathrm{Hg}$ ),
Table 2. Systemic hemodynamic parameters

\begin{tabular}{lcccc}
\hline & Baseline & End hypoxia & Predrug & \multicolumn{1}{l}{ End drug } \\
\hline Time & 0 min & $120 \mathrm{~min}$ & $240 \mathrm{~min}$ & $360 \mathrm{~min}$ \\
\hline \multicolumn{2}{l}{ Heart rate (beats/min) } & & \\
Sham & $173 \pm 11$ & $171 \pm 8$ & $195 \pm 11$ & $207 \pm 11$ \\
Control & $176 \pm 6$ & $208 \pm 11^{*}$ & $228 \pm 14$ & $206 \pm 6$ \\
D +E & $181 \pm 12$ & $208 \pm 14^{*}$ & $237 \pm 9^{*}$ & $269 \pm 12^{*+}$ \\
HD & $174 \pm 12$ & $233 \pm 7^{*}$ & $219 \pm 11$ & $247 \pm 20^{*+}$
\end{tabular}

Mean arterial pressure (MAP) $(\mathrm{mm} \mathrm{Hg})$

$\begin{array}{lllll}\text { Sham } & 77 \pm 3 & 63 \pm 4 & 54 \pm 2 & 48 \pm 2 \\ \text { Control } & 77 \pm 5 & 29 \pm 1^{*} & 48 \pm 4 & 41 \pm 2 \\ \text { D + E } & 74 \pm 4 & 26 \pm 1^{*} & 38 \pm 3^{*+} & 43 \pm 4 \\ \text { HD } & 75 \pm 4 & 29 \pm 1^{*} & 42 \pm 2^{*} & 44 \pm 1\end{array}$

Pulmonary arterial pressure (PAP) $(\mathrm{mm} \mathrm{Hg})$

$\begin{array}{lllll}\text { Sham } & 24 \pm 1 & 24 \pm 1 & 26 \pm 1 & 29 \pm 2 \\ \text { Control } & 25 \pm 2 & 30 \pm 2^{*} & 26 \pm 1 & 26 \pm 1 \\ \text { D+E } & 25 \pm 1 & 32 \pm 4^{*} & 26 \pm 1 & 29 \pm 2 \\ \text { HD } & 23 \pm 1 & 34 \pm 4^{*} & 24 \pm 1 & 26 \pm 2\end{array}$

PAP/MAP ratio

\begin{tabular}{lcccc} 
Sham & $0.32 \pm 0.04$ & $0.38 \pm 0.03$ & $0.50 \pm 0.04$ & $0.62 \pm 0.06$ \\
Control & $0.33 \pm 0.03$ & $1.03 \pm 0.03^{*}$ & $0.56 \pm 0.02$ & $0.65 \pm 0.04$ \\
$\mathrm{D}+\mathrm{E}$ & $0.34 \pm 0.02$ & $1.20 \pm 0.10^{*}$ & $0.69 \pm 0.09$ & \\
& & $0.70 \pm 0.08^{*}$ & & \\
HD & $0.31 \pm 0.02$ & $1.18 \pm 0.09^{*}$ & $0.57 \pm 0.02$ & $0.59 \pm 0.03$ \\
Cardiac index $(\mathrm{ml} / \mathrm{kg} / \mathrm{min})$ & & & \\
Sham & $224 \pm 30$ & $189 \pm 30$ & $199 \pm 26$ & $176 \pm 21$ \\
Control & $229 \pm 15$ & $78 \pm 5^{*}$ & $178 \pm 14$ & $176 \pm 12$ \\
$\mathrm{D}+\mathrm{E}$ & $205 \pm 28$ & $75 \pm 11^{*}$ & $137 \pm 22^{*}$ & $176 \pm 24$ \\
$\mathrm{HD}$ & $188 \pm 15$ & $70 \pm 10^{*}$ & $136 \pm 14^{*}$ & $177 \pm 15$ \\
\hline
\end{tabular}

Hypoxic-reoxygenated piglets treated with combination $\mathrm{D}+\mathrm{E}(n=7) ; \mathrm{HD}(n=7) ;$ or saline placebo (control, $n=7$ ). Sham-operated piglets had no hypoxia-reoxygenation $(n=6)$.

$\mathrm{D}+\mathrm{E}$, dopamine and epinephrine; $\mathrm{HD}$, high-dose dopamine.

${ }^{*} P<0.05$ vs. sham; ${ }^{\dagger} P<0.05$ vs. control.

pulmonary hypertension (pulmonary arterial pressure, PAP: $32 \pm 2 \mathrm{~mm} \mathrm{Hg})$, and reduced cardiac index (CI) $(36 \pm 2 \%$ of normoxic baseline) in comparison with sham-treated piglets (all $P<0.05$ ) (Table 2). Corresponding reductions in systemic oxygen delivery and consumption were also found (data not shown). H-R piglets showed significant decreases in common carotid, superior mesenteric, and renal oxygen perfusion $(22 \pm 2,15 \pm 1$, and $21 \pm 2 \%$ of respective normoxic baselines; $P<0.05$ vs. sham-treated piglets). Severe metabolic acidosis was present with significantly elevated plasma lactate levels in $\mathrm{H}-\mathrm{R}$ piglets (Table 1). No differences were found between $\mathrm{H}-\mathrm{R}$ groups regarding hemodynamic and biochemical parameters at the end of hypoxia.

\section{Reoxygenation}

After 10 min of $100 \%$ oxygen resuscitation $\left(\mathrm{PaO}_{2} 351 \pm 15 \mathrm{~mm}\right.$ $\mathrm{Hg}$ ), hemodynamic parameters improved significantly (data 
a

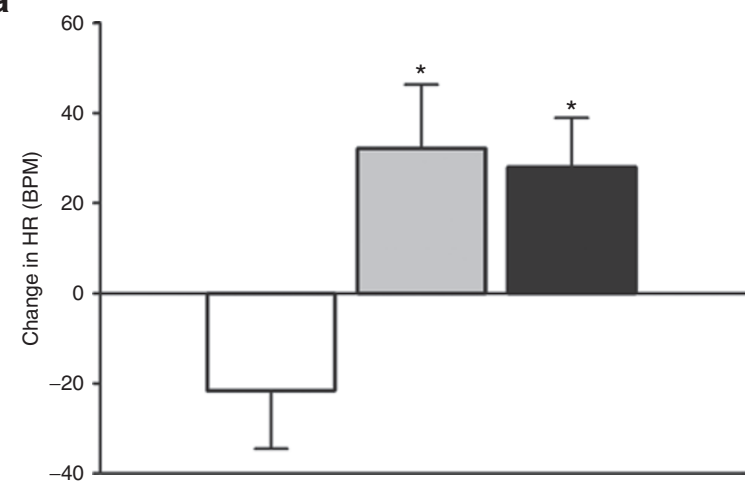

b

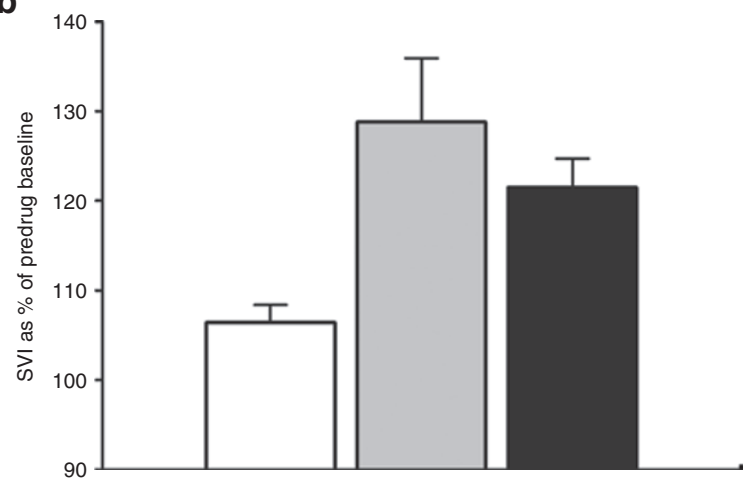

C

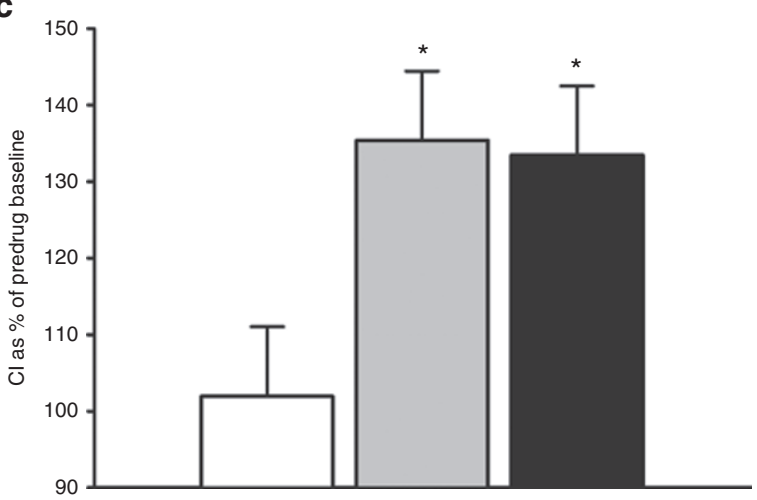

Figure 1. Changes in (a) heart rate (HR), (b) stroke volume index (SVI), and (c) cardiac index $(\mathrm{Cl})$ during the $2 \mathrm{~h}$ infusion of combination dopamine and epinephrine (gray) and high-dose dopamine (black) in hypoxia-reoxygenation (H-R) piglets in comparison with controls (white) ( $n=7 /$ group). $\mathrm{SVI}$ and $\mathrm{Cl}$ are expressed as mean percentage change over duration of infusion from predrug baseline. ${ }^{*} P<0.05 \mathrm{vs}$. control (two-way repeatedmeasures analysis of variance).

not shown) but then deteriorated over the following $2 \mathrm{~h}$. In $\mathrm{H}$-R piglets, MAP was $43 \pm 2 \mathrm{~mm} \mathrm{Hg}$ and CI was $73 \pm 4 \%$ of normoxic baseline (Table 2). Common carotid, superior mesenteric, and renal oxygen perfusion were $56 \pm 4,86 \pm 7$, and $79 \pm 8 \%$ of respective normoxic baselines after $2 \mathrm{~h}$ of reoxygenation and before medication delivery. All $\mathrm{H}-\mathrm{R}$ piglets had reduced serum bicarbonate and elevated plasma lactate (Table 1) and troponin I levels ( $P<0.05$ vs. sham-treated piglets). Of note, MAP of D + E piglets was significantly lower than that of control piglets $(P<0.05)$ (Table 2$)$.
Systemic/Pulmonary Hemodynamic Effects of Medications Both $\mathrm{HD}$ and $\mathrm{D}+\mathrm{E}$ groups increased heart rate over $2 \mathrm{~h}$ of infusion (vs. control, $P<0.05$ ) (Figure 1a and Table 2). The effect of these agents on stroke volume index was modest, with transient increases seen early during infusion (Figure $1 \mathrm{~b}$ ). The combined effect offered an increase in CI over the duration of infusion in $\mathrm{D}+\mathrm{E}$ and $\mathrm{HD}$ piglets (vs. control, $P<0.05$, Figure 1c). MAP decreased further in control piglets, whereas it increased in $\mathrm{D}+$ $\mathrm{E}$ and HD piglets (vs. control, $P<0.05$ ) (Figure 2a). There was no change in systemic vascular resistance during drug infusion (data not shown). PAP was unchanged during drug infusion (Figure $2 \mathrm{~b}$ ). $\mathrm{D}+\mathrm{E}$ piglets decreased PAP/MAP ratio during infusion relative to controls, which increased in $2 \mathrm{~h}(P<0.05)$ (Figure 2c).

\section{Regional Hemodynamic Effects of Medication Infusion}

Both $\mathrm{HD}$ and $\mathrm{D}+\mathrm{E}$ groups improved carotid artery flow index (CAFI) $(P<0.05$ and $P=0.05$ vs. control, respectively) and superior mesenteric artery flow index (both $P<0.05$ ) (Figure 3a). There were no associated changes in common carotid vascular resistance. However, mesenteric vascular resistance decreased significantly in the HD group $(P<0.05$ vs. control; $P=0.07$ vs. $\mathrm{D}+\mathrm{E}$ ) (Figure $3 \mathbf{b}$ ). There were no differences in renal artery flow index or renal vascular resistance among H-R groups during drug infusion.

Oxygen Delivery and Metabolism Effects of Medication Infusion $\mathrm{H}-\mathrm{R}$ piglets in the $\mathrm{HD}$ and $\mathrm{D}+\mathrm{E}$ groups had higher systemic oxygen delivery than that of controls $(P<0.05)$, with no effect on systemic oxygen consumption (Figure 4a). Common carotid and mesenteric oxygen delivery declined and remained unchanged, respectively, in control piglets. However, corresponding to the improvements in regional flow, HD piglets improved common carotid and superior mesenteric oxygen delivery ( $P<0.05$ vs. control), whereas $\mathrm{D}+\mathrm{E}$ group had modest improvements in these parameters $(P<0.1$ vs. control) (Figure $4 \mathbf{b}$ ). However, no effect was found on renal oxygen delivery. Plasma lactate levels of $\mathrm{H}-\mathrm{R}$ groups improved with no difference among groups over the $2 \mathrm{~h}$ infusion.

During the course of experimentation, plasma lactate levels correlated modestly with $\mathrm{CI}$ in all $\mathrm{H}-\mathrm{R}$ piglets $(r=-0.4, P<$ $0.05)$. Of note, the correlation was not significant in the control or HD groups, whereas significant correlation was found in the $\mathrm{D}+\mathrm{E}$ group $(r=-0.6, P<0.01)$. Systemic oxygen delivery correlated with plasma lactate $(r=-0.5, P<0.01)$, particularly in the $\mathrm{D}+\mathrm{E}$ and HD groups $(r=-0.6, P<0.01$ and $r=-0.5, P<$ 0.01 , respectively) but not in controls.

Tissue Oxidative Stress Markers, Plasma Troponin Levels, and Histologic Scores of H-R Injury

Ventricular lactate correlated with systemic oxygen delivery $(r=-0.6, P<0.01)$ in the H-R groups. Intestinal lactate correlated with superior mesenteric artery flow index and mesenteric oxygen delivery following $2 \mathrm{~h}$ of infusion $(r=-0.4, P=$ 0.05 and $r=-0.5, P=0.02$, respectively). Myocardial and intestinal tissue lactate, glutathione (GSH), and malondialdehyde 


\section{Articles | Manouchehriet al.}

a

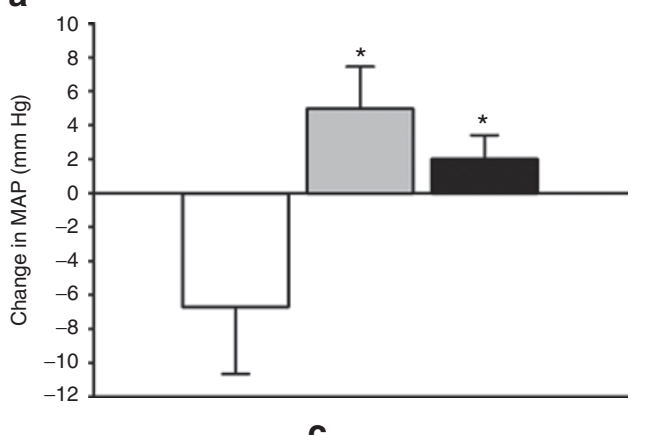

b

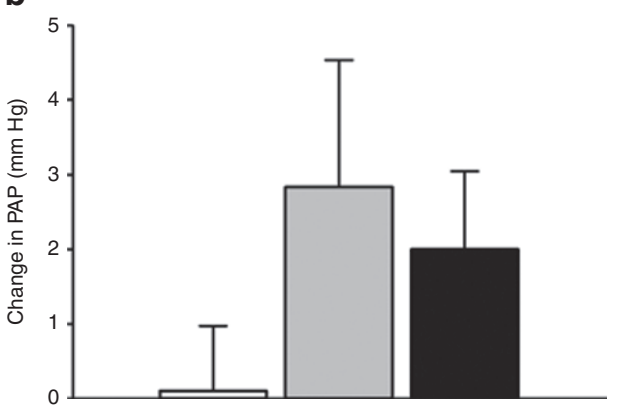

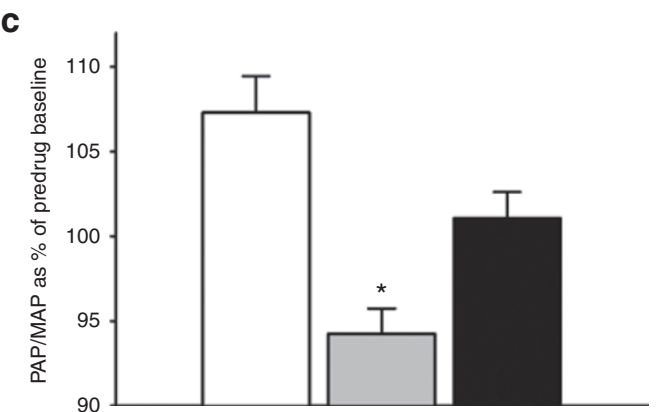

Figure 2. Changes in (a) mean arterial pressure (MAP), (b) pulmonary artery pressure (PAP), and (c) PAP/MAP ratio during the $2 \mathrm{~h}$ infusion of combination dopamine and epinephrine (gray) and high-dose dopamine (black) in hypoxia-reoxygenation (H-R) piglets in comparison with controls (white) ( $n=7 /$ group). PAP/MAP ratio is expressed as mean percentage change over duration of infusion from predrug baseline. ${ }^{*} P<0.05 \mathrm{vs}$. control (two-way repeatedmeasures ANOVA).

levels were not different among groups. Two cases each in the control and $\mathrm{HD}$ groups and three in the $\mathrm{D}+\mathrm{E}$ group at the time of necropsy had a colon that appeared to be ischemic. Despite these findings, there were no significant differences in histopathologic findings of H-R injury in the left ventricle and gut (data not shown). However, the degree of left-ventricle histologic injury did correlate modestly with plasma lactate levels at the end of experimentation $(r=0.4, P<0.05)$.

\section{DISCUSSION}

Following asphyxia of the neonate, shock with hypotension is often treated with vasoactive and/or inotropic medications $(17,18)$. On the basis of these findings in this swine model of neonatal $\mathrm{H}-\mathrm{R}$, monotherapy with $\mathrm{HD}$ and $\mathrm{D}+\mathrm{E}$ combination therapy have differential hemodynamic benefits in mesenteric and pulmonary circulations that need to be considered when asphyxiated neonates are at risk of compromised mesenteric perfusion and increased unbalanced pulmonary-systemic arterial pressure (PAP/MAP) ratio, respectively.

The treatment of piglets after H-R with either $\mathrm{HD}$ alone or $\mathrm{D}+\mathrm{E}$ combination improved myocardial function with chronotropic and transient inotropic effects. The improvement in CI with HD was not observed in previous studies using a similar model (9), nor were the predominant chronotropic effects of both regimens (J.E. Repetto et al., personal communication). This may be secondary to a significant protocol change with the use of only $30 \mathrm{~min}$ of pure oxygen resuscitation in lieu of a full hour. There were no differences in effect on systemic vascular resistance, further substantiating arguments against the detrimental vasoconstrictive effects of HD in neonatal H-R (19).
Similar to the findings of the only term neonate trial studying dopamine, MAP was improved in both treatment groups (20). Of note, at the doses studied, CI was improved without significant increase in afterload so as to worsen plasma lactate levels. Therefore, neither regimen, at least in the short period of therapy, causes adverse vasoconstrictive effects and impaired tissue perfusion. Further studies need to be conducted, however, to determine if this effect holds with prolonged infusion.

The failure to show differences in plasma lactate levels as well as in histopathological changes may be secondary to the limited $2 \mathrm{~h}$ time frame of study. The trend for increased lactate levels in the combination treatment piglets may indeed be proven valid if observations were to be carried further or higher doses of epinephrine infusion were administered (13). Increased plasma lactate, which is related to the epinephrineinduced increase in glycogenolysis and the associated increase in lactate production, has been observed in preterm neonates (11) and newborn piglets (21). Furthermore, safety from myocardial injury may need further investigation. The analysis of plasma troponin levels was complicated by nonsignificant but large intergroup differences before administration of the blinded medications. The measurement of brain natriuretic peptide levels in plasma may be useful, given that this parameter is often used clinically to assess myocardial performance after hypoxia and/or pulmonary hypertension. Nonetheless, with a $2 \mathrm{~h}$ infusion of either HD or D + E combination, there were no significant differences in myocardial lactate, GSH, or malondialdehyde levels among the groups.

Given that blood pressure and tissue perfusion are not synonymous, assessment of other parameters is required to 
a

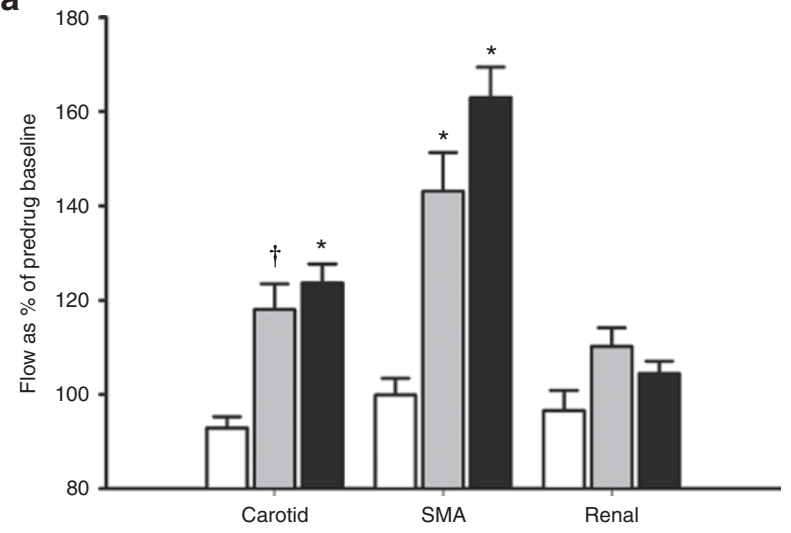

b

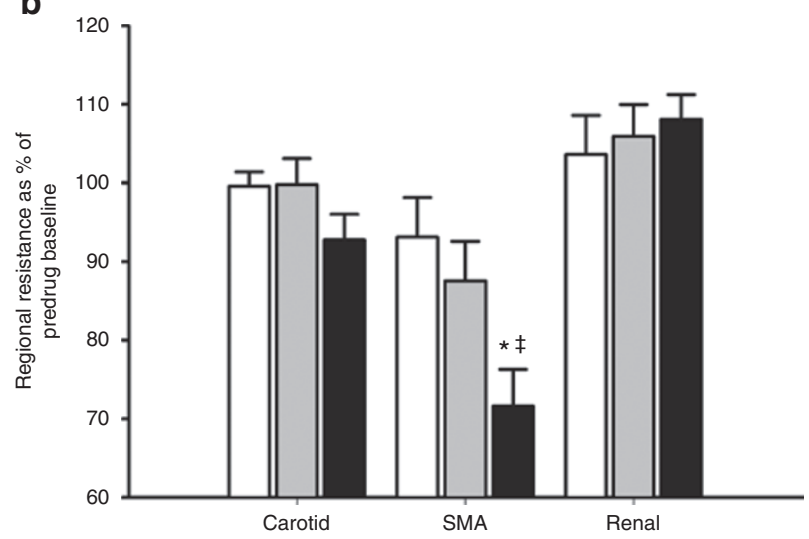

Figure 3. Changes in (a) carotid, superior mesenteric (SMA), and renal arterial flows, and (b) respective vascular resistance during the $2 \mathrm{~h}$ infusion of combination dopamine and epinephrine (gray) and high-dose dopamine (black) in hypoxia-reoxygenation (H-R) piglets in comparison with controls (white) ( $n=7 /$ group). Data are expressed as mean percentage change over duration of infusion from predrug baseline. ${ }^{*} P<0.05$ and ${ }^{\dagger} P=0.05$ vs. control; ${ }^{\ddagger} P=0.07$ vs. combination dopamine and epinephrine group (two-way repeated-measures ANOVA).

determine the status of asphyxiated neonates (22). In this study, we observed significant improvements in systemic oxygen delivery with either treatment, with no differences in oxygen consumption relative to controls. End-organ perfusion similarly improved, with benefits to carotid and mesenteric perfusion in both groups. Indeed, we demonstrated that improvements in mesenteric perfusion were associated with reduction of intestinal tissue lactate, a marker of anaerobic metabolism. However, treatment with HD alone significantly reduced mesenteric vascular resistance relative to controls and modestly reduced it relative to combination treatment. Previously, we found an increased mesenteric hemodynamics with a similar dose of dopamine in asphyxiated newborn piglets (9). Similar to our observations, a marked increase in mesenteric blood flow has been reported in normotensive neonatal piglets after dopamine infusion (21). Although the relationship between mesenteric perfusion and intestinal injury has not been clearly defined, ischemia is one of the pathophysiologi$\mathrm{cal}$ factors for necrotizing enterocolitis-like intestinal injury in asphyxiated term neonates $(23,24)$. Any improvement of mesenteric perfusion would be beneficial to neonates who are at a

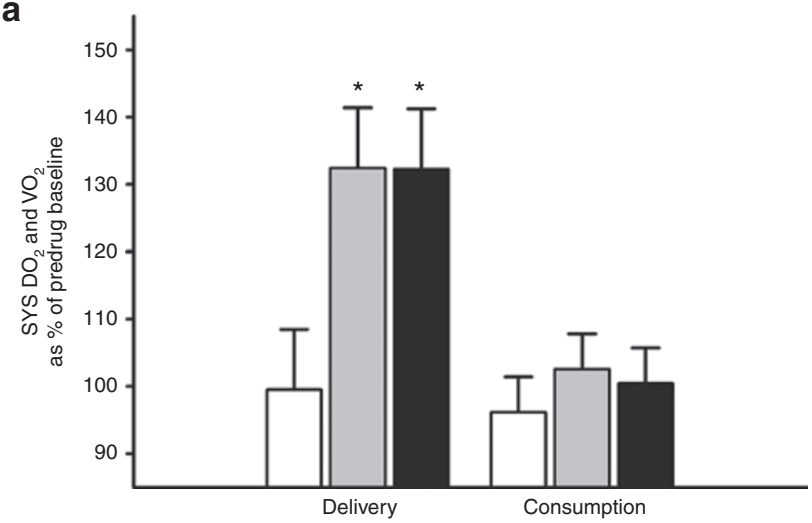

b

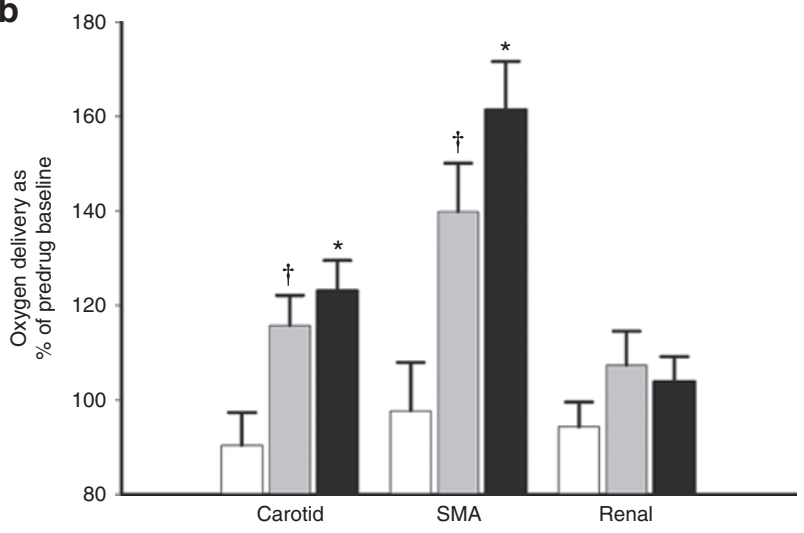

Figure 4. Changes in (a) systemic (SYS) oxygen delivery $\left(\mathrm{DO}_{2}\right)$ and consumption $\left(\mathrm{VO}_{2}\right)$, and (b) carotid, superior mesenteric, and renal $\mathrm{DO}_{2}$ during the $2 \mathrm{~h}$ infusion of combination dopamine and epinephrine (gray) and high-dose dopamine (black) in hypoxia-reoxygenation (H-R) piglets in comparison with controls (white) ( $n=7 /$ group). Data are expressed as mean percentage change over duration of infusion from predrug baseline. ${ }^{*} P<0.05$ and ${ }^{\dagger} P<0.1$ vs. control (two-way repeated-measures ANOVA). SMA, superior mesenteric artery.

risk for ischemic intestinal injury. The hemodynamic effect of HD treatment in mesenteric circulation should be studied in appropriately designed prospective trials in human neonates so that it can be considered when mesenteric perfusion and intestinal injury are of concern in neonates at risk for developing ischemic enterocolitis or intestinal vasculopathy.

The net effect observed on PAP/MAP ratio was different between the $\mathrm{HD}$ and $\mathrm{D}+\mathrm{E}$ combination groups, with the former stabilizing the ratio relative to placebo controls, in which it continued to increase. The addition of epinephrine reduced the ratio at the specified doses. This apparent "protective" effect of epinephrine in pulmonary-systemic hemodynamics is consistent with our previous observations of its differential effects on systemic and pulmonary vasculature in monotherapy experiments (25). These results may be relevant when we consider the combination of agents in managing neonates with persistent pulmonary hypertension or those at risk for developing pulmonary hypertension, given preferable effects on the risk for shunting of deoxygenated blood to the systemic circulation. However, appropriately designed prospective trials in neonates are required before the clinical relevance of these findings can safely be established. 


\section{Articles | Manouchehrietal.}

In this study, we did not observe any significant effect on renal perfusion with $\mathrm{HD}$ or $\mathrm{D}+\mathrm{E}$ administration in $\mathrm{H}-\mathrm{R}$ piglets. Neither was there a preferential vasodilatory increase in CAFI relative to $\mathrm{CI}$, although dopaminergic receptors are found in the vessels of the head and neck. In hemodynamically stable, normoxic newborn piglets, Nachar et al. (21) recently reported increases in blood flow in the renal, mesenteric, and common carotid circulations following escalating dosages of dopamine administration. Apart from differences in the drug administration protocol, the functionality of dopamine and adrenergic receptors after H-R may at least explain the lack of improved renal perfusion or preferentially increased CAFI.

The study is limited by the lack of improvement in systemic oxygen consumption following improvement in oxygen delivery in the drug treatment groups. One may infer that the piglets were not adequately stressed, given that oxygen metabolism was at maximal levels before drug infusion, offering no room for further improvement. This is different from prior studies using a similar $\mathrm{H}-\mathrm{R}$ model $(9,13,26)$ and may be related to the aforementioned differences in the reoxygenation protocol. Furthermore, this study uses a neonatal model to draw inferences regarding perinatal care, and therefore has significant limitations. As in other experimental studies, we used infusions of drug at fixed doses. Clinically, these agents tend to be titrated to effect. Indeed, despite of the lack of correlation between blood pressure and perfusion, it is common to use blood pressure as the target parameter of cardiovascular supportive therapy for critically ill neonates. Nonetheless, clinical practitioners are increasingly refraining from relying solely on MAP in the management of critically ill neonates with shock. Furthermore, upward titration of drug doses in pursuit of "normal" blood pressures may pose a concern given the potential for detrimental excessive vasoconstriction and impairment of tissue perfusion (18). Of note, we did not observe this phenomenon in $\mathrm{H}-\mathrm{R}$ piglets treated with $\mathrm{HD}$. The logistical implications with blinding and the relatively short duration of treatment will also limit the time for variable doses to effect a response, which was not possible in our short-term study. Furthermore, although the current drug protocol does not address if there is any priming effect by possible selective stimulation of dopaminergic and adrenergic receptors, we believe that our findings remain significant with good translational value with respect to the hemodynamic effects of $\mathrm{HD}$ vs. D + E administration. Indeed, the additional inotropic support is not uncommonly needed soon after starting dopamine at $5-10 \mathrm{mcg} / \mathrm{kg} / \mathrm{min}$. Furthermore, the in vivo nature of experiments limits a direct examination of possible vasoconstrictive action of these drugs. Vascular resistance is indicative of vascular resistance per second, although it has often been interpreted as a surrogate of vascular state.

The model is limited by differences between species, anesthetic effects, surgical instrumentation, clinical and experimental asphyxia, and resuscitation protocols. For example, although asphyxia is commonly associated with hypercapnia, severely hypoxic conditions of various cardiopulmonary pathologies are not uncommon in critically ill neonates and have similar clinical features to those of asphyxia with acidosis and hemodynamic compromise. Further hypotension in asphyxiated neonates is
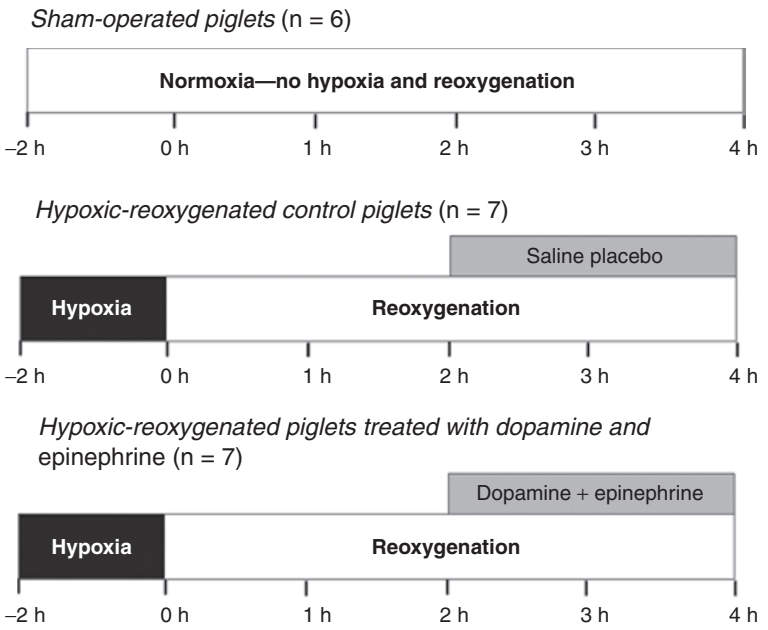

Hypoxic-reoxygenated piglets treated with high-dose dopamine $(\mathrm{n}=7)$

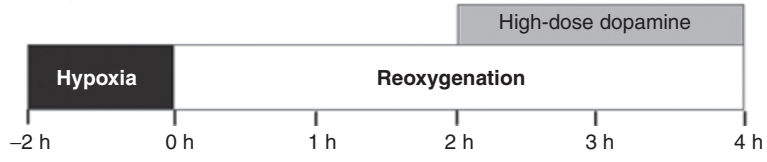

Figure 5. Experimental protocol.

related to a combination of cardiogenic and vasoplegic factors. On the basis of our previous observations in this model (26), the treatment with dobutamine does not provide significant vasoconstrictive effect. There is no clinical guideline in choosing the second inotrope. Nonetheless, studying the effects of dopamine and dobutamine combination will be interesting if different systemic hemodynamic effects would be found with direct and specific $\beta$-adrenergic stimulation. There are possible differences in adrenergic receptor functions between piglets and term human neonates. Differences in adrenoreceptor expression over the early life of the neonate as well as in the functionality after $\mathrm{H}-\mathrm{R}$ may complicate application of results in the perinatal setting of asphyxia. Therefore, it would be interesting albeit challenging to perform experiments on asphyxiatedreoxygenated animals in cardiogenic shock under the influence of adrenoceptor antagonists to distinguish mechanistically the systemic and regional hemodynamic effects.

In summary, in $\mathrm{H}-\mathrm{R}$ newborn piglets with severe cardiogenic shock and hypotension, despite a moderate dose of dopamine infusion, adding low-dose epinephrine or further increasing dopamine yield similar improvements in systemic hemodynamics (blood pressure and cardiac performance). However, the two regimens have differential effects on the pulmonary and mesenteric circulations. Further studies are needed to examine the peripheral circulations of the brain or other organs and to investigate the appropriate therapeutic approach in correcting the systemic and regional perfusion deficits in asphyxiated neonates after resuscitation.

\section{METHODS}

\section{Animals}

Mixed-breed (Duroc/Large White) 1-4-d-old piglets, weighing 1.5$2.5 \mathrm{~kg}$, were obtained from the university swine research unit on the morning of experimentation. The following protocol was approved 
by the university animal care and use committee and adheres to the Canadian Council on Animal Care guidelines.

\section{Anesthesia}

Isoflurane $(5 \%)$ was used to induce anesthesia, thereafter, titrated between $1 \%$ and $3 \%$ for maintenance. Following definitive airway placement and vascular access, inhalational anesthesia was discontinued. Mechanical ventilation was started with pressure control of $20 / 4 \mathrm{~cm} \mathrm{H}_{2} \mathrm{O}$ and rates of $18-20$ breaths/min (Sechrist Infant Ventilator Model IV-100; Sechrist Industries, Anaheim, CA) with fraction of inspired oxygen $\left(\mathrm{F}_{\mathrm{i}} \mathrm{O}_{2}\right)$ concentrations of $0.21-0.25$. Oxygen saturation was maintained at $88-100 \%$ (Nellcor, Hayward, CA). $\mathrm{F}_{\mathrm{i}} \mathrm{O}_{2}$ was measured using a MiniOx III oxygen monitor (Catalyst Research, Owings Mills, MD). Throughout experimentation, piglets were maintained at a temperature of $38.5-40{ }^{\circ} \mathrm{C}$ using both a heating underpad and an overhead warmer. Anesthesia was maintained with a combination of intravenous midazolam $(0.2-1 \mathrm{mg} / \mathrm{kg} / \mathrm{h})$ for sedation and fentanyl $(5-20 \mathrm{mcg} / \mathrm{kg} / \mathrm{h})$ for analgesia. Pancuronium $(0.05-$ $0.1 \mathrm{mg} / \mathrm{kg} / \mathrm{h}$ ) was administered for paralysis during surgery. Boluses of fentanyl $(10 \mathrm{mcg} / \mathrm{kg})$ and acepromazine $(0.25 \mathrm{mg} / \mathrm{kg})$ were used as needed; $5 \%$ dextrose $(20 \mathrm{ml} / \mathrm{h})$ and $0.9 \%$ normal saline $(4 \mathrm{ml} / \mathrm{h})$ were infused with boluses of lactated Ringer's solution given as indicated.

\section{Surgical Instrumentation}

The right femoral artery and vein were exposed through a groin incision. The femoral vein was cannulated with a 5-Fr dual-lumen catheter (Sherwood Medical, St. Louis, MO) advanced $13-15 \mathrm{~cm}$ to the right atrium to administer fluid and medications and measure central venous pressure. A 5-Fr single-lumen catheter was then inserted into the femoral artery and advanced $5 \mathrm{~cm}$ to the infrarenal aorta for MAP monitoring. Next, via a transverse neck incision, a $3.5 \mathrm{~mm}$ inner diameter endotracheal tube was inserted through a tracheotomy to allow mechanical ventilation.

The left common carotid artery was encircled with a calibrated 2-mm flow probe (2SS; Transonic Systems, Ithaca, NY). A left subcostal incision was used for exposure of the retroperitoneum, where the left kidney was reflected anteriorly and the superior mesenteric artery was exposed. Here, a 3-mm flow probe (3SB) was placed and the left renal artery was then encircled with a similar 2-mm (2SB) flow probe. Following a left anterior thoracotomy, the pericardium was opened and the ductus arteriosus was ligated. The pulmonary artery was then cannulated using a 20-gauge angiocatheter (Insyte-W, Becton Dickinson Infusion Therapy Systems, Sandy, UT) for PAP monitoring. A 6-mm flow probe (6SB) was then placed around the main pulmonary artery to measure blood flow as a surrogate for cardiac output.

\section{Stabilization and Monitoring}

After instrumentation, piglets received a bolus of $10 \mathrm{ml} / \mathrm{kg}$ lactated Ringer's solution and were allowed to recover for $60 \mathrm{~min}$. Arterial $\mathrm{PCO}_{2}$ of $35-45 \mathrm{~mm} \mathrm{Hg}$ was maintained throughout experimentation. Heart rate and blood pressures were monitored continuously using a Hewlett Packard 78834A monitor (Hewlett Packard, Palo Alto, CA). Hemodynamic readings were digitized at a sampling rate of $24 / \mathrm{s}$ and recorded in a personal computer equipped with custom Asyst programming software (Data Translation, Mississauga, Ontario, Canada).

\section{$\mathrm{H}-\mathrm{R}$ Protocol}

Piglets were block randomized to either a surgical sham group or one of three H-R groups (Figure 5). Sham-operated piglets (sham, $n=6$ ) were maintained at $\mathrm{F}_{\mathrm{i}} \mathrm{O}_{2}$ of $0.21-0.25$ throughout the experimentation period (6h). H-R piglets underwent normocapnic alveolar hypoxia, through addition of inhaled nitrogen, reducing $\mathrm{F}_{\mathrm{i}} \mathrm{O}_{2}$ to $0.10-0.15$ for $2 \mathrm{~h}$. $\mathrm{PaO}_{2}$ was maintained at $20-40 \mathrm{~mm} \mathrm{Hg}$ during hypoxia to produce cardiac dysfunction and hypotension as previously described (26-28). Subsequently, piglets were resuscitated with $100 \%$ oxygen for 30 min and kept at $\mathrm{F}_{\mathrm{i}} \mathrm{O}_{2} 0.21-0.25$ for the remainder of experimentation $(3.5 \mathrm{~h})$ to maintain normoxia in these sick animals. A $10 \mathrm{ml} / \mathrm{kg}$ bolus of lactated Ringer's solution was administered during reoxygenation before drug infusion.

At $2 \mathrm{~h}$ of reoxygenation, $\mathrm{H}-\mathrm{R}$ piglets received infusions of either placebo $(0.9 \%$ saline solution-control) or study drug at a constant rate in a blinded, randomized fashion ( $n=7 /$ group). Study drug groups included the following: HD group: dopamine $(20 \mathrm{mcg} / \mathrm{kg} /$ min; Baxter, Toronto, Ontario, Canada), or D + E combination group: dopamine $(10 \mathrm{mcg} / \mathrm{kg} / \mathrm{min})$ and epinephrine $(0.1 \mathrm{mcg} / \mathrm{kg} / \mathrm{min}$; Erfa Canada, Westmount, Quebec, Canada). A laboratory technician prepared drug solutions in standard volume syringes for blinding purposes. The dosages were derived in part from previous studies in a similar swine model of neonatal $\mathrm{H}-\mathrm{R}$, and the epinephrine dosage was decided in consideration of its concomitant administration with dopamine $(9,13)$.

\section{Hemodynamic and Oxygen Measurements}

Heart rate, MAP, PAP, and central venous pressure were recorded continuously and analyzed at set intervals from normoxic baseline through hypoxia and reoxygenation and at every $30 \mathrm{~min}$ of placebo or drug treatment. The data were averaged as means over a 2 -min recording at each time point. In addition, systemic and pulmonary arterial blood was sampled every $15 \mathrm{~min}$ during hypoxia and at $10,30,60,120,150,180,210$, and $240 \mathrm{~min}$ after reoxygenation for blood gas analysis using an ABL 700 blood gas analyzer and OSM3 Hemoximeter (Radiometer, Copenhagen, Denmark).

\section{Biochemical Analysis and Histopathology}

Arterial samples were analyzed for lactate using the ABL 700 analyzer, and plasma was stored at $-80{ }^{\circ} \mathrm{C}$. Plasma levels of porcine cardiacspecific troponin-I at baseline, $2 \mathrm{~h}$ of reoxygenation, and $2 \mathrm{~h}$ of drug treatment were analyzed using enzyme-linked immunosorbent assay (no. 2010-4, Life Diagnostics, West Chester, PA).

At the end of experimentation, piglets were killed using intravenous pentobarbital $(100 \mathrm{mg} / \mathrm{kg})$. Necropsy was performed immediately for retrieval of left-ventricular tissue and terminal ileum. A small sample was stored in $10 \%$ formalin solution, and the remaining tissue was snap-frozen in liquid nitrogen and stored at $-80{ }^{\circ} \mathrm{C}$. The formalinpreserved specimen was then processed for hematoxylin and eosin staining. Histologic findings were interpreted without knowledge of treatment allocation using previously described scoring systems for H-R injury $(29,30)$.

For measurement of tissue lactate, frozen myocardial and intestinal tissue were crushed and homogenized in $6 \%$ perchloric acid $/ 0.5 \mathrm{mmol} / \mathrm{l}$ ethyleneglycol-bis(baminoethylether)- $N, N 9$-tetraacetic acid on ice. Samples were centrifuged and supernatant collected. A total of $5 \mathrm{~mol} / \mathrm{l}$ of potassium carbonate was then added slowly in a 1:10 $\mu \mathrm{l}$ of supernatant ratio. Following precipitation for $30 \mathrm{~min}$, samples were again centrifuged and supernatant was collected for nicotinamide adenine dinucleotide enzyme-coupled colorimetric microplate assay. After the addition of glycylglycine buffer, nicotinamide adenine dinucleotide, double-distilled water, glutamate-pyruvate transaminase, and lactate dehydrogenase, absorbance was read at $340 \mathrm{~nm}$ using a microplate spectrophotometer (Spectramax 190; Molecular Devices, Sunnyvale, CA).

Measurement of tissue GSH was performed using a commercially available GSH assay kit (Cayman Chemical, Ann Arbor, MI). Frozen tissue was crushed and homogenized in buffer containing $0.2 \mathrm{~mol}$ 2-( $N$-morpholino)ethanesulphonic acid, $50 \mathrm{mmol} / \mathrm{l}$ phosphate, and $1 \mathrm{mmol} / \mathrm{l}$ EDTA at $\mathrm{pH}$ 6-7. Following centrifugation, supernatant was deproteinated with $10 \%$ metaphosphoric acid and $4 \mathrm{~mol} / \mathrm{l}$ triethanolamine. Colorimetric microplate assay was then performed after the addition of GSH reductase, glucose-6-phosphate dehydrogenase, oxidized nicotinamide adenine dinucleotide phosphate, and 5,5'-dithiobis-2-nitrobenzoic acid. Absorbance was read at $405 \mathrm{~nm}$ after $25 \mathrm{~min}$ using the aforementioned spectrophotometer. To measure oxidized GSH, reduced GSH was derivatized to oxidized GSH using 2 -vinylpyridine. The assay was then carried out using this sample. Oxidative status of GSH was determined through interpretation of the oxidized GSH/GSH ratio.

For measurement of tissue malondialdehyde, frozen tissues were homogenized 1:10 in phosphate-buffered saline and fluorescence assays were compared with standard concentrations of malondialdehyde as described by Ohkawa et al. (31).

\section{Statistics}

Data were analyzed using SigmaPlot v11 software (Systat Software, San Jose, CA). ANOVA (one-way and two-way repeated-measures) 
was used where appropriate for parametric data, with post hoc pairwise analysis by the Student-Newman-Keuls test. For nonparametric data, the Kruskal-Wallis test was performed with Dunn's method for post hoc intergroup comparison. For practical interpretation of drug effect after infusion, percentage change respective to predrug baseline was used to analyze data during the drug infusion phase. Correlations between variables were performed with the Pearson moment or Spearman test as appropriate. Significance was defined as $P<0.05$. Results are expressed as mean \pm SEM.

\section{STATEMENT OF FINANCIAL SUPPORT}

The project was funded by an operating grant (MOP53116) from the Canadian Institutes of Health Research. N.M. received support from the Clinician Investigator Program of the Royal College of Physicians and Surgeons of Canada. P.-Y.C. was an investigator of the Canadian Institutes of Health Research and the Alberta Heritage Foundation for Medical Research.

\section{REFERENCES}

1. World Health Organization. Newborns: no longer going unnoticed. In: The World Health Report 2005: Make Every Mother and Child Count. Geneva, Switzerland: WHO Press, World Health Organization, 2005:79-101.

2. Martín-Ancel A, García-Alix A, Gayá F, Cabañas F, Burgueros M, Quero J. Multiple organ involvement in perinatal asphyxia. J Pediatr 1995;127:78693.

3. Evans N. Which inotrope for which baby? Arch Dis Child Fetal Neonatal Ed 2006;91:F213-20.

4. Alonso-Spilsbury M, Mota-Rojas D, Villanueva-García D, et al. Perinatal asphyxia pathophysiology in pig and human: a review. Anim Reprod Sci 2005;90:1-30.

5. Cheung PY, Johnson ST, Obaid L, Chan GS, Bigam DL. The systemic, pulmonary and regional hemodynamic recovery of asphyxiated newborn piglets resuscitated with $18 \%, 21 \%$ and $100 \%$ oxygen. Resuscitation 2008;76: 457-64.

6. Fanaroff JM, Fanaroff AA. Blood pressure disorders in the neonate: hypotension and hypertension. Semin Fetal Neonatal Med 2006;11: $174-81$.

7. Subhedar NV. Treatment of hypotension in newborns. Semin Neonatol 2003;8:413-23.

8. Seri I. Systemic and pulmonary effects of vasopressors and inotropes in the neonate. Biol Neonate 2006;89:340-2.

9. Obaid L, Johnson ST, Emara M, Bigam DL, Cheung PY. Epinephrine versus dopamine to treat shock in hypoxic newborn pigs resuscitated with 100\% oxygen. Shock 2008;29:262-8.

10. Voelckel WG, Lurie KG, McKnite S, et al. Comparison of epinephrine and vasopressin in a pediatric porcine model of asphyxial cardiac arrest. Crit Care Med 2000;28:3777-83.

11. Valverde E, Pellicer A, Madero R, Elorza D, Quero J, Cabañas F. Dopamine versus epinephrine for cardiovascular support in low birth weight infants: analysis of systemic effects and neonatal clinical outcomes. Pediatrics 2006;117:e1213-22.

12. Heckmann M, Trotter A, Pohlandt F, Lindner W. Epinephrine treatment of hypotension in very low birthweight infants. Acta Paediatr 2002;91: 566-70.
13. Cheung PY, Abozaid S, Al-Salam Z, Johnson S, Li Y, Bigam D. Systemic and regional hemodynamic effects of high-dose epinephrine infusion in hypoxic piglets resuscitated with 100\% oxygen. Shock 2007;28:491-7.

14. Seri I. Cardiovascular, renal, and endocrine actions of dopamine in neonates and children. J Pediatr 1995;126:333-44.

15. Paradisis M, Evans N, Kluckow M, Osborn D. Randomized trial of milrinone versus placebo for prevention of low systemic blood flow in very preterm infants. J Pediatr 2009;154:189-95.

16. Pellicer A, Valverde E, Elorza MD, et al. Cardiovascular support for low birth weight infants and cerebral hemodynamics: a randomized, blinded, clinical trial. Pediatrics 2005;115:1501-12.

17. Piazza AJ. Postasphyxial management of the newborn. Clin Perinatol 1999;26:749-65, ix.

18. Seri I. Inotrope, lusitrope, and pressor use in neonates. J Perinatol 2005;25:Suppl 2:S28-30.

19. Seri I, Evans J. Controversies in the diagnosis and management of hypotension in the newborn infant. Curr Opin Pediatr 2001;13:116-23.

20. DiSessa TG, Leitner M, Ti CC, Gluck L, Coen R, Friedman WF. The cardiovascular effects of dopamine in the severely asphyxiated neonate. J Pediatr 1981;99:772-6.

21. Nachar RA, Booth EA, Friedlich P, et al. Dose-dependent hemodynamic and metabolic effects of vasoactive medications in normotensive, anesthetized neonatal piglets. Pediatr Res 2011;70:473-9.

22. Seri I. Cardiovascular support in the preterm: treatments in search of indications. J Pediatr 2007;150:e31-3; author reply e33.

23. Ito Y, Doelle SM, Clark JA, Halpern MD, McCuskey RS, Dvorak B. Intestinal microcirculatory dysfunction during the development of experimental necrotizing enterocolitis. Pediatr Res 2007;61:180-4.

24. Nankervis CA, Giannone PJ, Reber KM. The neonatal intestinal vasculature: contributing factors to necrotizing enterocolitis. Semin Perinatol 2008;32:83-91.

25. Cheung PY, Barrington KJ. The effects of dopamine and epinephrine on hemodynamics and oxygen metabolism in hypoxic anesthetized piglets. Crit Care 2001;5:158-66.

26. Al-Salam Z, Johnson S, Abozaid S, Bigam D, Cheung PY. The hemodynamic effects of dobutamine during reoxygenation after hypoxia: a doseresponse study in newborn pigs. Shock 2007;28:317-25.

27. Johnson ST, Bigam DL, Emara M, et al. N-acetylcysteine improves the hemodynamics and oxidative stress in hypoxic newborn pigs reoxygenated with $100 \%$ oxygen. Shock 2007;28:484-90.

28. Joynt C, Bigam DL, Charrois G, Jewell LD, Korbutt G, Cheung PY. Doseresponse effects of milrinone on hemodynamics of newborn pigs with hypoxia-reoxygenation. Intensive Care Med 2008;34:1321-9.

29. Rose AG, Opie LH, Bricknell OL. Early experimental myocardial infarction. Evaluation of histologic criteria and comparison with biochemical and electrocardiographic measurements. Arch Pathol Lab Med 1976;100:516-21.

30. Park PO, Haglund U, Bulkley GB, Fält K. The sequence of development of intestinal tissue injury after strangulation ischemia and reperfusion. Surgery 1990;107:574-80.

31. Ohkawa H, Ohishi N, Yagi K. Assay for lipid peroxides in animal tissues by thiobarbituric acid reaction. Anal Biochem 1979;95:351-8. 